\title{
Regulation of UDP-Glucuronosyltransferase 2B15 by miR-331- $5 p$ in Prostate Cancer Cells Involves Canonical and Noncanonical Target Sites $\underline{\underline{s}}$
}

\author{
Dhilushi D. Wijayakumara, Peter I. Mackenzie, Ross A. McKinnon, Dong Gui Hu, ${ }^{1}$ \\ and Robyn Meech ${ }^{1}$ \\ Department of Clinical Pharmacology and Flinders Centre for Innovation in Cancer, Flinders University College of Medicine and \\ Public Health, Flinders Medical Centre, Bedford Park, South Australia, Australia
}

Received October 25, 2017; accepted November 30, 2017

\begin{abstract}
UGT2B15 is an important androgen-metabolizing UDP-glucuronosyltransferase (UGT) and the mechanisms controlling its expression are of considerable interest. Recent studies showed that miR-376c regulates UGT2B15 in prostate cancer cells via a canonical target site in the $3^{\prime}$ untranslated region ( $\left.3^{\prime} U T R\right)$. The UGT2B15 3'UTR also contains a canonical miR-331-5p target site; previous work indicated that deleting this site reduced, but did not abolish, the ability of miR-331-5p to repress a luciferase reporter carrying the UGT2B15 3'UTR. We report here the discovery and characterization of a second, noncanonical miR331-5p target site in the UGT2B15 $3^{\prime}$ UTR. miR-331-5pmediated repression of a UGT2B15 3'UTR-reporter was partly inhibited by mutating either of the two miR-331-5p target sites separately, but completely abolished by mutating the two sites simultaneously, indicating that the two sites act cooperatively. miR-331-5p mimics significantly reduced both UGT2B15 mRNA levels and glucuronidation activity in prostate cancer cells,
\end{abstract}

confirming that the native transcript is a miR-331-5p target. Transfection of either miR-331-5p or miR-376c mimics repressed the activity of the UGT2B15 3'UTR-reporter; however, cotransfection of both microRNAs (miRNAs) further reduced activity, indicating cooperative regulation by these two miRNAs. A significant negative correlation between miR-331 and UGT2B15 mRNA levels was observed in a tissue RNA panel, and analysis of The Cancer Genome Atlas (TCGA) hepatocellular carcinoma data set provided further evidence that miR-331 may play an important role in regulation of UGT2B15 in vivo. There was no significant correlation between miR-331 and UGT2B15 mRNA levels in the TCGA prostate adenocarcinoma cohort, which may reflect the complexity of androgen-mediated regulation in determining UGT2B15 levels in prostate cancer. Finally, we show that miR-331-5p does not regulate UGT2B17, providing the first evidence for a posttranscriptional mechanism that differentially regulates these two important androgen-metabolizing UGTs.

\section{Introduction}

Human UDP-glucuronosyltransferases (UGTs) are a superfamily of enzymes that catalyze glucuronidation, a conjugation reaction that is critical for detoxification and clearance of a large number of endogenous and exogenous small lipophilic compounds including carcinogens, drugs, and biologically active signaling molecules (e.g., steroid hormones) (Mackenzie et al., 2005). Human UGTs are categorized into four subfamilies according to their sequence similarity,

This study was supported by the National Health and Medical Research Council of Australia [Grant ID1020931 (to P.I.M.) and Grant ID1085410 (to P.I.M., R.A.M., and R.M.)]. The project was also supported by funding from the Flinders Medical Centre Foundation.

R.A.M. is a Cancer Council/SA Health Beat Cancer Professorial Chair. During the preparation period, P.I.M. was a National Health and Medical Research Council Senior Principal Research Fellow; R.M. was an Australian Research Council Future Fellow.

${ }^{1}$ D.G.H. and R.M. contributed equally to this work.

https://doi.org/10.1124/jpet.117.245936.

S This article has supplemental material available at jpet.aspetjournals.org. namely, UGT1, UGT2, UGT3, and UGT8 (Mackenzie et al., 1997, 2005). In humans, glucuronidation of androgens (e.g., testosterone and dihydrotestosterone) and their metabolites (e.g., androsterone and androstane- $3 \alpha, 17 \beta$-diol) is carried out by three UGTs, namely, UGT2B7, UGT2B15, and UGT2B17 (Turgeon et al., 2001). The androgen/androgen receptor (AR) signaling pathway plays a pivotal role in human prostate growth and function; however, excessive androgen signaling within the prostate contributes to prostate cancer development and progression (Kaarbø et al., 2007). UGT2B15 and UGT2B17 are expressed in the prostate and their glucuronidation of the active androgens (e.g., testosterone and dihydrotesterone) renders them inactive as ligands for the AR, thus representing the end of androgen signaling within the prostate (Chouinard et al., 2008). These two UGTs are also expressed in liver where they conjugate and promote elimination of androgens, thus reducing circulating androgen levels. Consistent with their important functions in androgen signaling, altered UGT2B15 and UGT2B17 activity is related to the risk of developing androgen-dependent prostate

ABBREVIATIONS: AR, androgen receptor; bp, base pair; LIHC, liver hepatocellular carcinoma; miRNA, microRNA; miR-neg, negative control miRNA; 4-MU, 4-methylumbelliferone; nt, nucleotide; PRAD, prostate adenocarcinoma; TCGA, The Cancer Genome Atlas; UGT, UDPglucuronosyltransferase; UTR, untranslated region. 
diseases such as prostate cancer (Turgeon et al., 2001; Heinlein and Chang, 2004; Chouinard et al., 2007).

Mechanisms that control the intraprostatic and hepatic levels of UGT2B15 and UGT2B17 are still not well defined. The transcriptional regulation of UGT2B15 and UGT2B17 in the prostate has been the subject of many studies using AR-positive prostate cancer cell lines (e.g., LNCaP). These studies have shown that UGT2B15 and UGT2B17 are downregulated by a number of endogenous and exogenous compounds including androgens (e.g., testosterone and dihydrotestosterone), farnesoid $\mathrm{X}$ receptor activators (e.g., chenodeoxycholic acid), and calcitriol ( $1 \alpha$,25-dihydroxyvitamin $\mathrm{D}_{3}$ ) (Chouinard et al., 2007; Bao et al., 2008; Kaeding et al., 2008a,b; Hu et al., 2014).

MicroRNAs (miRNAs), 21-25 nucleotide (nt)-long, noncoding small RNAs, control gene expression at the post-transcriptional level through translational repression and/or mRNA degradation (He and Hannon, 2004; Shukla et al., 2011). Each miRNA has a 6-nt seed sequence that is considered to be important for recognition of its target mRNA. The corresponding 6-nt region in the mRNA target, which makes direct contact with the miRNA seed sequence, is called the seed site. The miRNA seed sequence is located between nucleotides 2 and 7 from the $5^{\prime}$ terminus of a miRNA. The seed site (in the mRNA) is located between nucleotides 2 and 7 from the $3^{\prime}$ terminus of the miRNA recognition site in the target $\mathrm{mRNA}$.

Canonical miRNA target sites in mRNAs have a seed site that has a perfect Watson-Crick base pairing to its cognate miRNA seed sequence. Noncanonical miRNA target sites contain a seed site that mispairs to the seed sequence of its cognate miRNA by at least $1 \mathrm{nt}$. At canonical sites, the miRNA/mRNA duplex formation mainly relies on Watson-Crick base pairing via the seed sequence ( $5^{\prime}$ pairing), whereas at noncanonical sites the miRNA/mRNA duplex formation primarily occurs via an extensive base pairing at the miRNA center (middle pairing) or $3^{\prime}$ end ( $3^{\prime}$ pairing). Recent studies have shown that about $50 \%$ of miRNA target sites are noncanonical sites (Shin et al., 2010; Loeb et al., 2012; Helwak et al., 2013; Martin et al., 2014). Posttranscriptional regulation of several $U G T$ genes by miRNAs via canonical miRNA target sites in various cellular contexts have been recently reported (Dluzen et al., 2014, 2016; Wijayakumara et al., 2015, 2017; Margaillan et al., 2016). For example, we and others recently reported post-transcriptional regulation of UGT2B15 and UGT2B17 by miR-376c in prostate cancer LNCaP cells via a canonical target site that is conserved in their 3' untranslated regions (UTRs) (Wijayakumara et al., 2015; Margaillan et al., 2016). Margaillan et al. (2016) also reported a putative canonical miR-331-5p target site at the UGT2B15 3'UTR and showed that deleting the seed site of this canonical miR-331-5p site reduced the ability of miR-331-5p to repress the activity of a luciferase reporter carrying the UGT2B15 3'UTR. In the present study, we discovered a second noncanonical miR-331$5 p$ site in the UGT2B15 3'UTR and showed that the two sites mediate cooperative regulation of UGT2B15. miR-331-5p was also shown to target endogenous UGT2B15 expression and thus activity levels; however, it did not alter UGT2B17 mRNA levels. UGT2B15 and miR-331-5p levels are inversely correlated in RNA samples from multiple tissues, and in a liver cancer data set, but not in a prostate cancer data set. Overall, our data suggest that miR-331-5p and miR-376c are important regulators of UGT2B15 levels, but may play varying roles in different tissues; moreover, miR-331-5p mediates differential regulation of the UGT2B15 and UGT2B17 mRNAs.

\section{Materials and Methods}

miRNA Mimics and Human Tissue Total RNAs. Synthetic mimics corresponding to hsa-miR-331-5p, hsa-miR-376c, and a negative control miRNA (miR-neg) were purchased from Shanghai GenePharma (Shanghai, China). Total RNA samples representing a human multitissue panel were purchased from Thermofisher (Ambion FirstChoice Human Total RNA Survey Panel, Thermofisher, Waltham, MA).

Cell Transfection, RNA Extraction, and Reverse-Transcriptase Quantitative Real-Time Polymerase Chain Reaction. The prostate cancer LNCaP cells were maintained in RPMI 1640 Medium (Gibco/Life Technologies, Grand Island, NY) containing 5\% (v/v) fetal bovine serum at $37^{\circ} \mathrm{C}$ in $5 \% \mathrm{CO}_{2}$ atmosphere. Prior to transfection, cells were cultured in phenol red-free RPMI $1640 \mathrm{Me}$ dium (Invitrogen, Carlsbad, CA) supplemented with 5\% dextrancoated charcoal-stripped fetal bovine serum for 32 hours and plated in six-well plates at approximately $1 \times 10^{6}$ cells per well. Following overnight culture ( $\sim 16$ hours), cells at $\sim 70 \%$ confluence were transfected with miR-331-5p mimics or miR-neg in triplicate at $30 \mathrm{nM}$ using $8 \mu \mathrm{l}$ Lipofectamine 2000 (Invitrogen). Total RNA was extracted 24 hours post-transfection using TRIzol (Invitrogen) and the target genes were quantified using reverse-transcriptase quantitative real-time polymerase chain reactions in a RotorGene 3000 instrument (Corbett Research, NSW, Australia) as previously reported ( $\mathrm{Hu}$ and Mackenzie, 2009, 2010; Hu et al., 2010). Quantification of miR-331-5p and miR-U6 small nuclear-2 RNA (termed RNU6-2) was performed as previously reported (Balcells et al., 2011; Wijayakumara et al., 2015). The mRNA levels of UGT2B15 and glyceraldehyde-3-phosphate dehydrogenase relative to $18 \mathrm{~S}$ rRNA and the miR-331-5p levels relative to that of RNU6-2 levels were quantified using the $2^{-\Delta \Delta \mathrm{Ct}}$ method (Livak and Schmittgen, 2001). The miRNA-specific primers used are listed in Table 1.

Generation of pGL3 Reporter Constructs and Mutagenesis. The pGL3 reporter constructs containing the UGT2B15 3'UTR (pGL3/2B15/UTR) or UGT2B17 3'UTR (pGL3/2B17/UTR) cloned downstream from the luciferase gene were recently reported (Wijayakumara et al., 2015). Using the pGL3/2B15/UTR construct as the template and the QuickChange site-directed mutagenesis kit (Stratagene, La Jolla, CA), the seed site of the canonical miR-331-5p target site (5'-AUACCUA-3 ${ }^{\prime}$ ) was either mutated to $5^{\prime}$-CGCAACA-3' or deleted. The mutated (pGL3/2B15/UTR/miR-331-5p/MT) and deleted (pGL3/2B15/ UTR/miR331-5p/delMT) reporter constructs are shown in Fig. 1B.

Deletion analysis was used to discover the noncanonical miR-331$5 p$ target site. Eight different segments of the UGT2B15 3'UTR were amplified using pGL3/2B15/UTR as the template and Phusion hotstart high-fidelity DNA polymerase (Themo Fisher Scientific, Pittsburgh, PA). The segments of the UGT2B15 3'UTR were numbered relative to the nucleotide $\mathrm{G}$ (set as position 0 ) of the stop codon TAG (NM_001076.3). The amplicons were cloned into the XbaI site of the pGL3 promoter vector downstream from the luciferase gene, generating eight pGL3 reporter constructs: pGL3/2B15/1-75, pGL3/ 2B15/1-150, pGL3/2B15/1-225, pGL3/2B15/1-314, pGL3/2B15/1-384, pGL3/2B15/244-314, pGL3/2B15/244-384, and pGL3/2B15/244-454 (Fig. 4A).

To further define the noncanonical miR-331-5p target site predicted in the 75-150 base pair (bp) region of the UGT2B15 3'UTR, we created mutations at site A (MT1 and MT2) and site B (MT3), which were predicted by RNAhybrid (Krüger and Rehmsmeier, 2006) as potential miR-331-5p target sites and also at three other positions as negative controls (MT4, MT5, and MT6) using the pGL3/2B15/1-150 construct as the template and the QuikChange site-directed mutagenesis kit (Stratagene). The sequence of each mutation is shown in Fig. 4B. To investigate the cooperativity of the two miR-331-5p sites, the canonical (site 1) and noncanonical (site 2) miR-331-5p target sites were mutated separately (Fig. 5A, MT A, MT B, and MT D) and simultaneously (Fig. 5A, MT C and MT E) using the pGL3/2B15/UTR reporter construct as the template. The identities of all of the aforementioned 
TABLE 1

Primers used in this study for mutagenesis, cloning, and RT-qPCR $\left(5^{\prime}-3^{\prime}\right)$

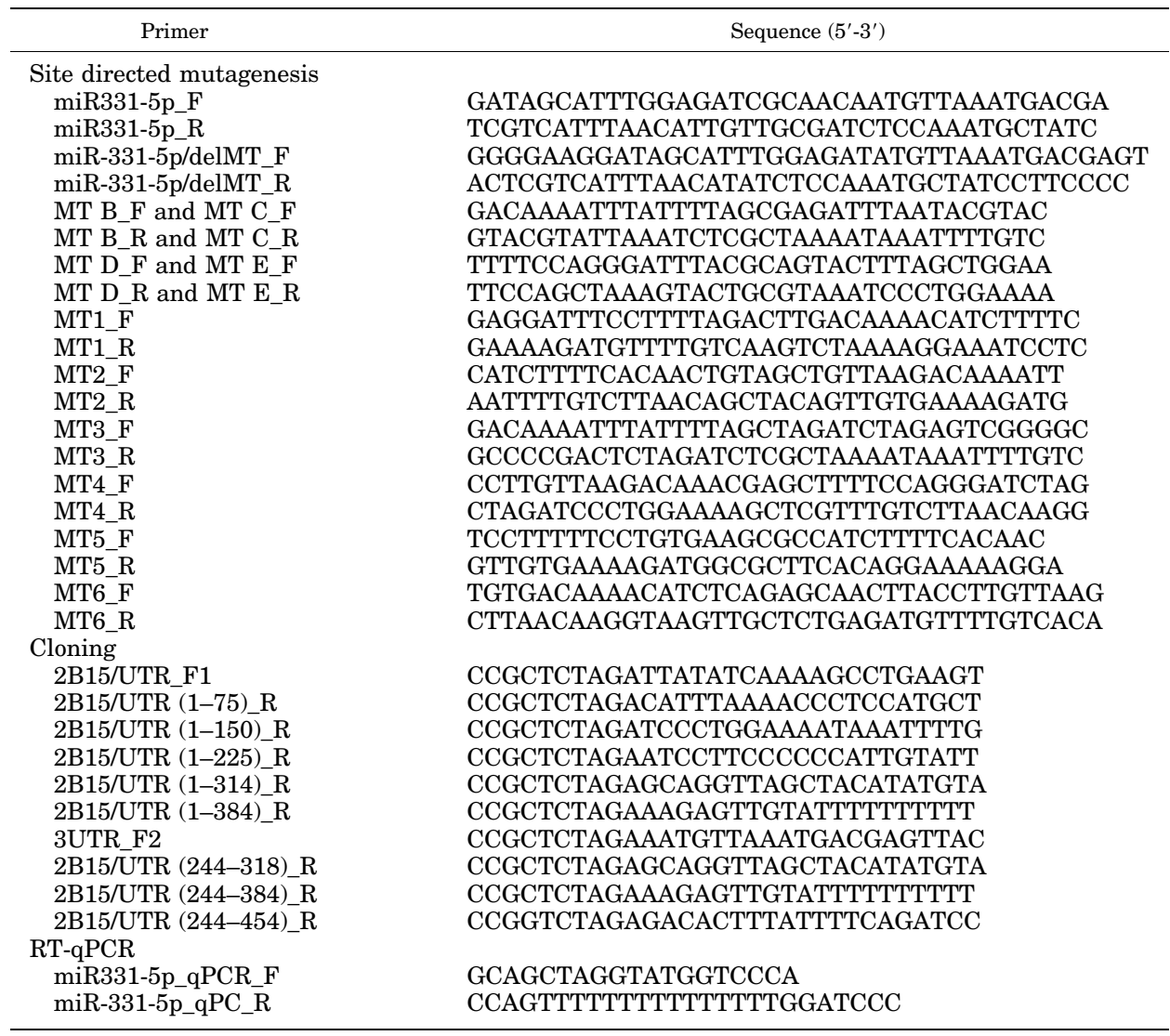

RT-qPCR, reverse-transcriptase quantitative real-time polymerase chain reaction.

constructs were confirmed by DNA sequencing. Primers used for cloning and mutagenesis are listed in Table 1.

Luciferase Reporter Assays. LNCaP cells were plated in 96-well plates at approximately $1.25 \times 10^{5}$ cells per well, cultured overnight, and then transfected with $100 \mathrm{ng}$ of reporter plasmid, $0.8 \mathrm{ng}$ of pRL-null vector and $4.5 \mathrm{pmol}$ of either miR-331-5p mimics or miRneg. Then, 24 hours post-transfection cells were lysed in passive lysis buffer, and subjected to Dual-Luciferase Reporter Assay according to the manufacturer's instructions (Promega, Madison, WI). Firefly and Renilla luciferase activities were measured on a Packard TopCount luminescence and scintillation counter (PerkinElmer Life and Analytical Sciences, Waltham, MA). The firefly luciferase activity was normalized to the Renilla luciferase activity and presented relative to that of the empty pGL3-promoter vector (set as a value of 1 ).

4-Methylumbelliferone Glucuronidation Assays. 4-Methylumbelliferone (4-MU) glucuronidation assays were performed using whole cell lysates of LNCaP cells transfected with miR-331-5p mimics or miR-neg as previously reported (Wijayakumara et al., 2015). Briefly, the 200- $\mu$ l glucuronidation assay mixture that contained $100 \mathrm{mM}$ potassium phosphate $\mathrm{pH} 7.4,4 \mathrm{mM} \mathrm{MgCl}_{2}, 400 \mu \mathrm{M}$ 4-MU, $225 \mu \mathrm{g}$ lysate, and $5 \mathrm{mM}$ UDP-glucuronic acid was incubated for 2 hours at $37^{\circ} \mathrm{C}$ in a shaking water bath. Previous studies indicate that the assay remains linear at this time point. After the addition of $2 \mu \mathrm{l}$ of $70 \%$ perchloric acid, samples were kept on ice for a minimum of 10 minutes and centrifuged at $5000 \mathrm{~g}$ for 10 minutes at $4^{\circ} \mathrm{C}$. Then, $60 \mu \mathrm{l}$ of the supernatant fraction was analyzed by high-performance liquid chromatography using an Agilent 1100 series instrument (Agilent Technologies, Sydney, Australia) as previously described (Uchaipichat et al., 2004). Concentrations of 4-MU glucuronide in samples were quantified using a standard curve of 4-MU glucuronide prepared over the concentration ranges of $0.5-10 \mu \mathrm{M}$. Human
UGT2B15 supersomes (Corning, Corning NY) were included as controls in these assays.

Analyses of Prostate Adenocarcinoma (PRAD) and Hepatocellular Carcinoma Data Sets. Transcriptome profiling RNA sequencing and miRNA sequencing data from liver hepatocellular carcinomas (LIHCs) and PRADs were downloaded from The Cancer Genome Atlas (TCGA) data portal (https://gdc-portal.nci.nih.gov/) and analyzed as previously reported (Wijayakumara et al., 2017). Briefly, the RNA sequencing expression data from 371 LIHC samples and 498 PRAD samples were represented in the form of high-throughput sequencing counts. Genes (protein coding and noncoding) with a mean of less than 10 counts were discarded; the counts of the remaining genes were normalized using the upper quantile normalization method. Correlation analyses between the expression levels of UGT and miRNA gene sets were conducted using the Spearman rank method and plots were drawn using the $\mathrm{R}$ statistical package (https:// cran.r-project.org/; R Foundation for Statistical Computing, Vienna, Austria).

Statistical Analysis. Statistical analyses of all data were performed using GraphPad Prism 6 software (GraphPad, La Jolla, CA), with a two-tailed Student's independent $t$ test. Correlation between UGT2B15 mRNA and miR-331-5p levels in the human tissue RNA panel was analyzed using Spearman's correlation. Values of $P<0.05$ were considered statistically significant.

\section{Results}

A Canonical miR-331-5p Target Site Is Present in the UGT2B15 3'UTR. TargetScan (release 7.0: August 2015; http://www.targetscan.org/vert_70/docs/help.html) predicted a 

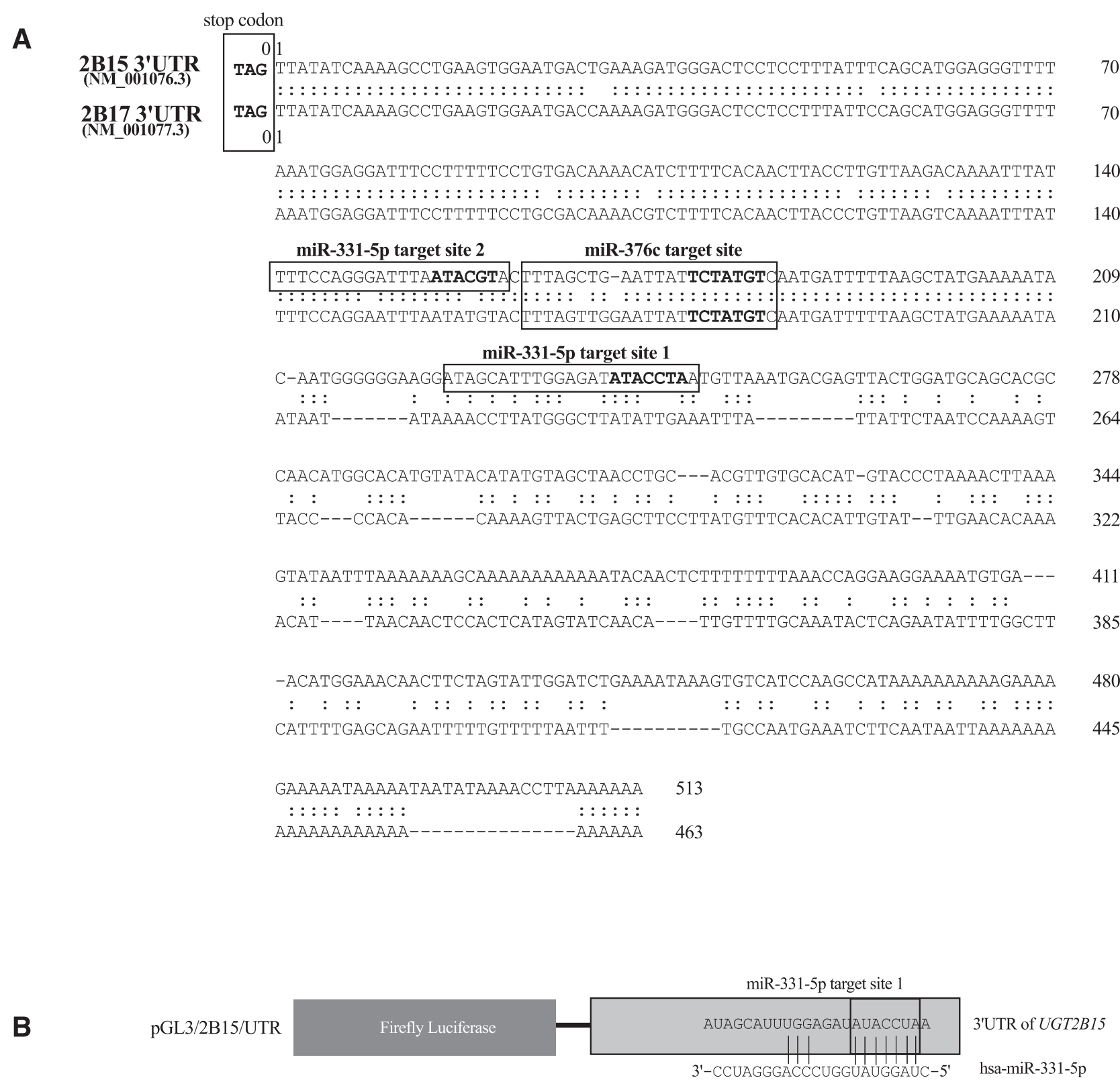

pGL3/2B15/UTR/miR-331-5p/MT

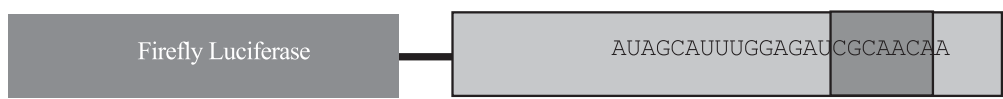

3'UTR of $U G T 2 B 15$

pGL3/2B15/UTR/miR-331-5p/delMT
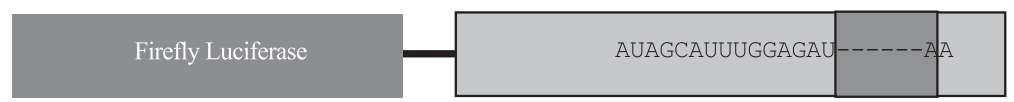

3'UTR of UGT2B15

Fig. 1. Predicted miRNA target sites in the UGT2B15 and UGT2B17 3'UTRs. (A) Sequence alignment of UGT2B15 (NM_001076.3) and UGT2B17 (NM_001077.3) 3'UTR regions. Boxes indicate UGT2B15 miR-331-5p target sites 1 and 2, and the miR-376c target site that is conserved and functional in both UGT2B15 and UGT2B17 3'UTRs. (B) Schematic representation of firefly luciferase reporter constructs containing the full-length UGT2B15 3'UTR bearing a wild-type miR-331-5p site 1 (pGL3/2B15/UTR), a seed-site mutated miR-331-5p site 1 (pGL3/2B15/UTR/miR-331-5p/MT), or a seed-site deleted miR-331-5p site 1 (pGL3/2B15/UTR/miR-331-5p/delMT).

miR-331-5p target site at nucleotides 224-249 of the UGT2B15 3'UTR (Fig. 1A). This site pairs perfectly to miR-331-5p at nucleotides 2-7 (seed pairing) and nucleotides 13-15 (3'-pairing), and hence is considered a canonical miR-331-5p target site (Lewis et al., 2005; Grimson et al., 2007). Although this site has been previously reported, whether miR-331-5p is a regulator of endogenous UGT2B15 mRNA and enzymatic activity has not yet been assessed.

To investigate regulation of UGT2B15 by miR-331-5p we chose prostate cancer LNCaP cells because they express high levels of UGT2B15 mRNA (Wijayakumara et al., 2015) and extremely low levels of miR-331-5p (data not shown). 
Transfection of miR-331-5p mimics into LNCaP cells significantly reduced UGT2B15 mRNA levels compared with those in miR-neg-transfected cells (Fig. 2A). Control glyceraldehyde3 -phosphate dehydrogenase mRNA levels were not significantly altered by miR-331-5p mimics (Fig. 2B). Next, we transfected LNCaP cells with miR-331-5p mimics or the miRneg control and assessed changes in 4-MU glucuronidation activity as previously reported (Wijayakumara et al., 2015). As shown in Fig. 2C, miR-331-5p mimics significantly reduced 4-MU glucuronidation activity compared with miR-neg mimics. Thus, miR-331-5p reduces both UGT2B15 mRNA levels and activity. To assess the role of the predicted canonical miR-331-5p target site in regulation of UGT2B15, we generated luciferase reporters carrying the wild-type UGT2B15 3'-UTR (pGL3/2B15/UTR), and reporters with either mutation or deletion at the seed site of the miR-331-5p target site within the $3^{\prime} \mathrm{UTR}$ (pGL3/2B15/UTR/ miR331-5p/MT or pGL3/2B15/UTR/miR331-5p/delMT). These reporters were transfected with miR-331-5p mimics or miR-neg into LNCaP cells. As shown in Fig. 3, miR-331-5p mimics significantly repressed the activity of the reporter carrying the wild-type UGT2B15 3'UTR by $\sim 70 \%$. Interestingly, this repression was only partially abrogated by mutation (Fig. 3A) or deletion (Fig. 3B) at the seed site of the miR-331-5p target site. While these results confirmed that miR-331-5p can repress the UGT2B15 3'UTR at least partially through the canonical miR331-5p site as reported by Margaillan et al. (2016); it also suggested that there are other regions of the $3^{\prime}$ UTR involved in miR-331-5p activity.

A Noncanonical miR-331-5p Target Site Is Present in the UGT2B15 3'UTR. Our observation (Fig. 3) that miR331-5p mimics repressed pGL3/2B15/UTR reporters in which the canonical miR-331-5p target site was deleted/mutated strongly suggested that the $3^{\prime}$ UTR might contain additional miR-331-5p target site(s). Unfortunately, such site(s) could not be readily identified using bioinformatic approaches since most miRNA target site prediction programs including TargetScan rely on a seed sequence match between a miRNA and its target mRNAs for target site prediction, and hence are unable to predict noncanonical target sites with a mismatched seed site or without a seed site (Lewis et al., 2005; Grimson et al., 2007; Shin et al., 2010; Loeb et al., 2012; Cloonan, 2015). To identify additional noncanonical miR-331-5p target site(s), we used an unbiased deletion analysis approach. A series of pGL3 reporter constructs carrying different segments of the UGT2B15 3'UTR (i.e., nt1-75, nt1-150, nt1-225, nt1-314, nt1384, nt1-454, nt244-318, nt244-384, and nt244-454) were transfected with either miR-331-5p mimics or miR-neg into LNCaP cells (Fig. 4A). As expected, miR-331-5p mimics significantly reduced the activity of two constructs carrying the canonical miR-331-5p target site (nt1-314 and nt1-384) and had no impact on the activity of another three constructs lacking the canonical miR-331-5p target site (nt244-318, nt244-384, and nt244-454). The miR-331-5p mimics significantly reduced the activities of two other constructs (nt1-150 and nt1-225) lacking the canonical miR-331-5p target site and did not affect the activity of the construct (nt1-75), thus suggesting the existence of a miR-331-5p target site within nt75-150.

Analysis of the 75-150 bp region of the UGT2B15 3'UTR by RNAhybrid, a miRNA target site prediction program previously shown to be able to predict noncanonical target sites (Krüger and Rehmsmeier, 2006), predicted two energetically favorable duplex formation sites between miR-331-5p and UGT2B15 mRNA at nucleotides 76-122 (termed site A: -18.8 $\mathrm{kcal} / \mathrm{mol}$ ) and at nucleotides 141-150 (termed site B: -15.7 $\mathrm{kcal} / \mathrm{mol}$ ) (Fig. 4B). To assess whether miR-331-5p could target these two sites, we created mutations in the nt1-150 construct that either disrupted (Fig. 4B, MT1, MT2, and MT3) or did not affect (Fig. 4B, MT4, MT5, and MT6) the predicted duplex formation sites. Transfection of these mutated constructs with
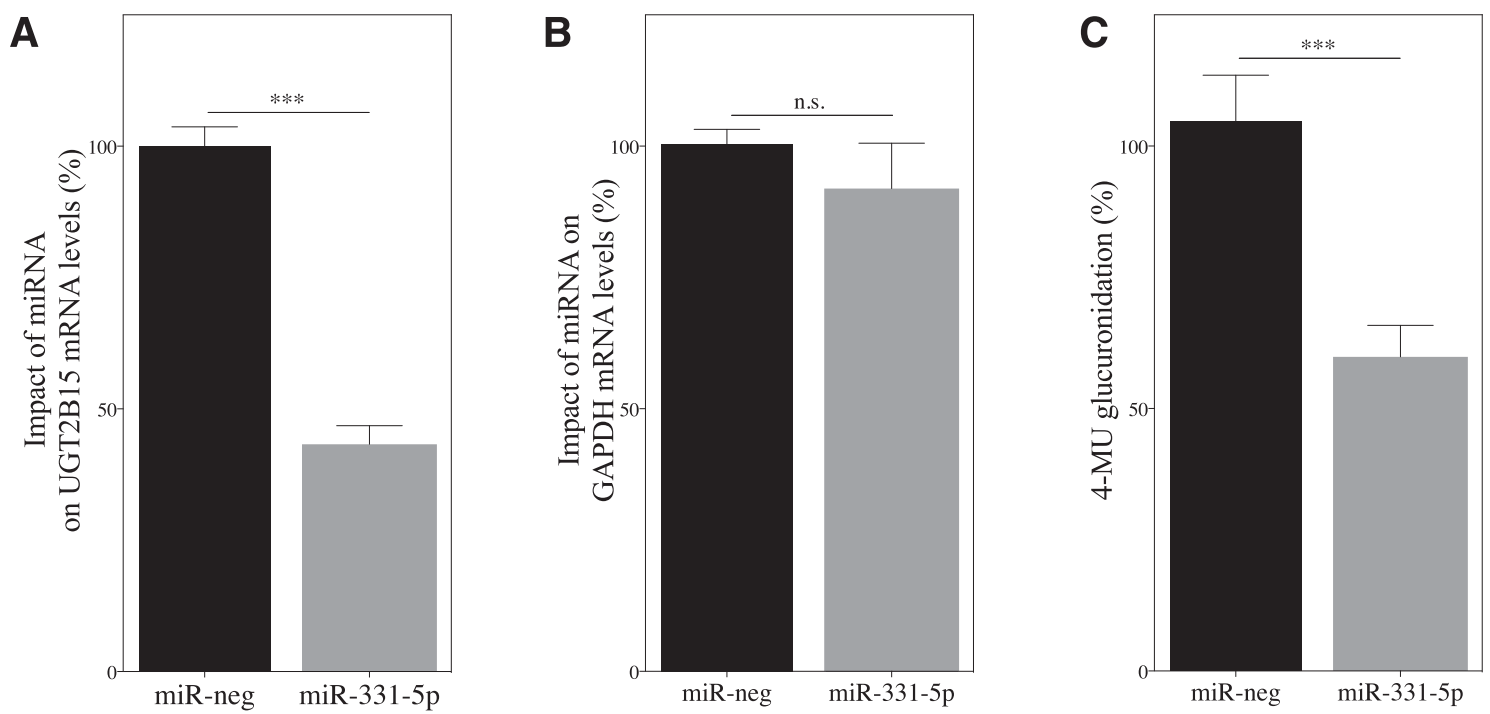

Fig. 2. miR-331-5p reduces both UGT2B15 mRNA levels and enzymatic activity in LNCaP cells. (A and B) LNCaP cells were transfected with miR-331$5 \mathrm{p}$ or miR-neg at $30 \mathrm{nM}$ in triplicate; RNA was extracted and subjected to reverse-transcriptase quantitative real-time polymerase chain reaction to measure target mRNA levels. After normalization to $\beta$-actin mRNA levels, the mRNA levels of UGT2B15 (A) or glyceraldehyde-3-phosphate dehydrogenase (GAPDH) (B) in cells transfected with miR-331-5p are presented relative to those in miR-neg-transfected cells (set at a value of 100\%). (C) The 4-methylumbelliferone (4-MU) glucuronidation activity in LNCaP cells transfected with miR-331-5p mimics is presented relative to those in miR-neg-transfected cells (set as a value of $100 \%$ ). Data shown are mean \pm S.E.M. from at least two independent experiments performed in triplicate. $* * * P<0.001$. 


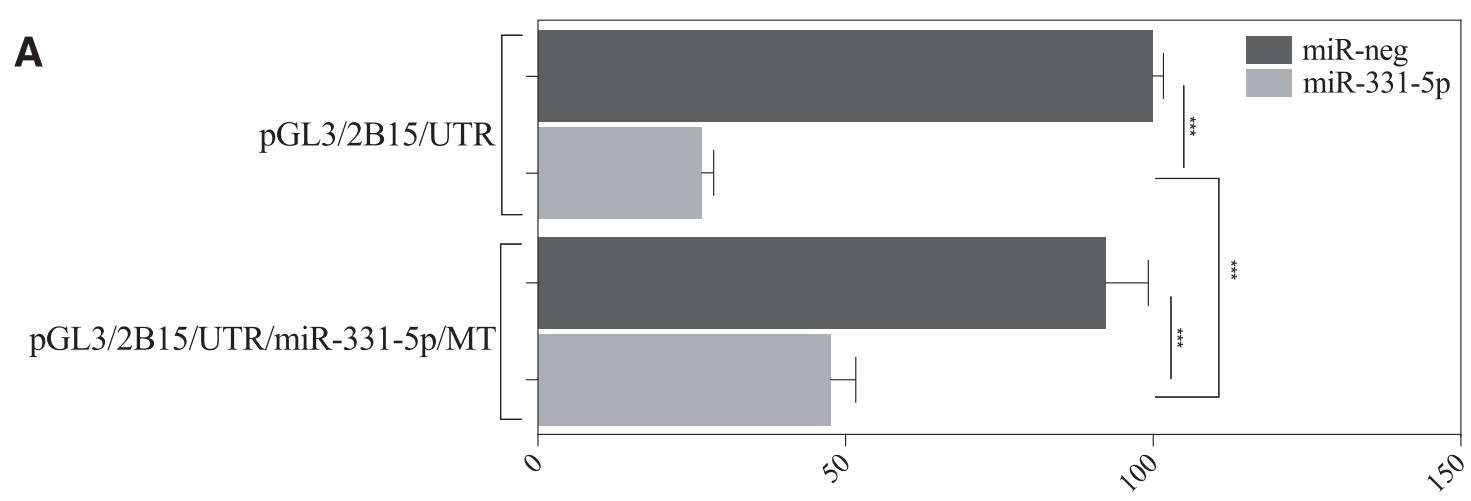

Relative luciferase activity $(\%)$

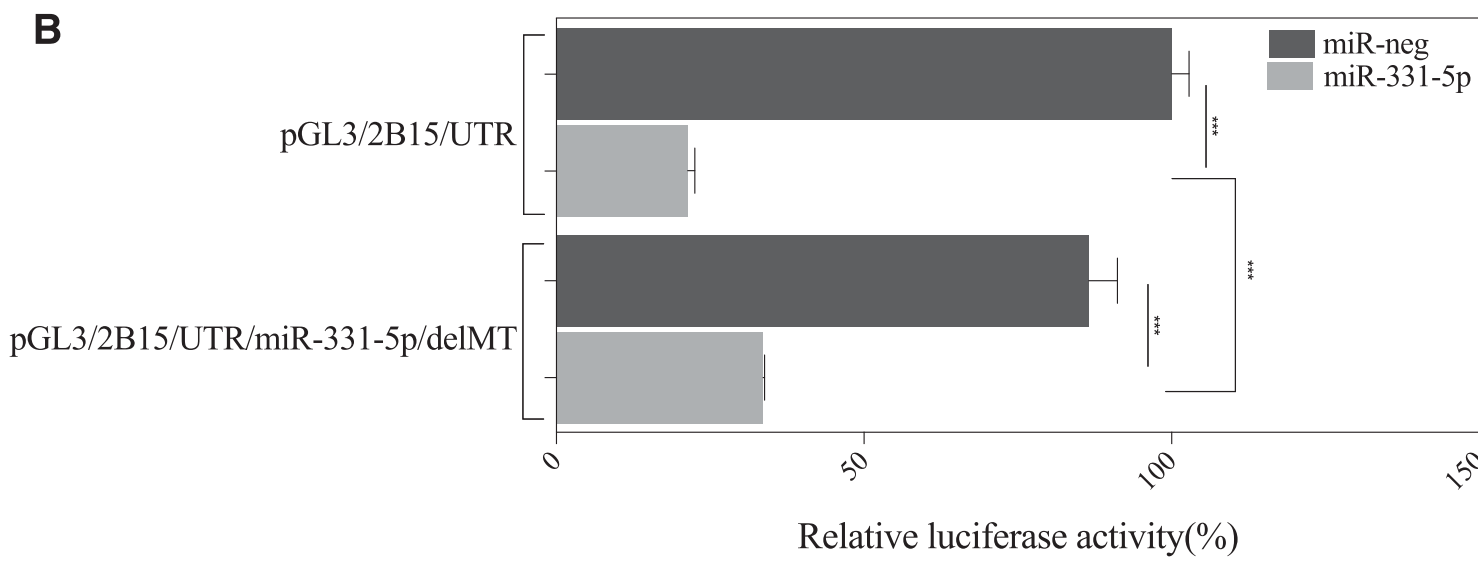

Fig. 3. miR-331-5p mimics reduce the activity of a pGL3 firefly luciferase reporter construct carrying the UGT2B15 3'UTR via a canonical target site (site 1) in LNCaP cells. Reporter constructs containing the full-length UGT2B15 3'UTR with wild-type, seed site mutated (A), or seed site deleted (B) miR-331-5p site 1 were transfected with miR-neg or miR-331-5p into LNCaP cells. Luciferase reporter assays were performed as described in Materials and Methods. The activity of the reporter constructs was normalized to the activity of the pRL-null reference vector and is presented relative to the activities of the empty pGL3-promoter vector (set at a value of 100\%). Data shown are mean \pm S.E.M. from at least two independent experiments performed in quadruplicate. ${ }^{* * *} P<0.0001$.

either miR-331-5p mimics or miR-neg into LNCaP cells showed that only MT3 completely abolished the ability of miR-331-5p mimics to repress reporter activity (Fig. 4C), thus indicating that site $B$ is an active miR-331-5p target site. Site B consists of $10 \mathrm{nt}$, eight of which pair with miR-331-5p at its $3^{\prime}$ end, suggesting extensive $3^{\prime}$ pairing between the miRNA and target site (Fig. 4B). Analysis of the 454-bp full-length UGT2B15 3'UTR by RNAhybrid predicted a 22-nt miR-331-5p target site containing the 10-nt site B at nucleotides 141-162 (-22.1 $\mathrm{kcal} / \mathrm{mol}$ ) (Fig. 4B). RNAhybrid predicted Watson-Crick pairing at $16 \mathrm{nt}$ between this site and miR-331-5p, including pairing in the seed site but with a 1-bp mismatch; hence, we define this as a noncanonical miR-331-5p target site. For simplicity, we termed this noncanonical miR-331 site as miR-331-5p site 2 and the canonical seed-matched miR-331-5p site as miR331-5p site 1 (Fig. 1A).

Cooperative Regulation of UGT2B15 3'UTR by miR331-5p via the Two miR-331-5p Target Sites. To assess any cooperativity between the two miR-331-5p target sites, we mutated the two sites separately or in combination (Fig. 5A, MT C) in the pGL3/UTR/UGT2B15 nt1-454 luciferase reporter; the mutants were cotransfected with either miR-331-
$5 p$ mimics or miR-neg into LNCaP cells. The mutation in site 1 was located in the seed site (Fig. 5A, MT A), whereas two mutations were generated in site 2: a mutation disrupting the 3 'pairing (Fig. 5A, MT B and MT C), and a mutation in the seed site (Fig. 5A, MT D and MT E). The ability of miRNA-331$5 \mathrm{p}$ mimics to repress reporter activity was partially but significantly reduced by mutation of the two sites separately; however, it was completely abrogated when the two sites were mutated simultaneously (Fig. 5B). This demonstrates a synergistic regulation of UGT2B15 by the two miR-331-5p sites. Of note, this cooperativity was not seen when the miR331-5p site was mutated at the 1-bp mismatched seed site (Fig. 5B, MT E) but only when mutated at the 3 ' sequence (Fig. $5 \mathrm{~B}, \mathrm{MT} \mathrm{C}$ ), suggesting that the $3^{\prime}$ pairing plays a critical role at miRNA/mRNA duplex formation at the UGT2B15 miR-331$5 \mathrm{p}$ site 2 .

Cooperative Regulation of UGT2B15 3'UTR by miR-331-5p and miR-376c. The recently reported miR$376 \mathrm{c}$ target site is located between the two miR-331-5p sites characterized in Fig. 5 (Wijayakumara et al., 2015; Margaillan et al., 2016) (Fig. 1A). To examine whether miR-376c and miR-331-5p cooperatively regulate the UGT2B15 3'UTR, we 
A
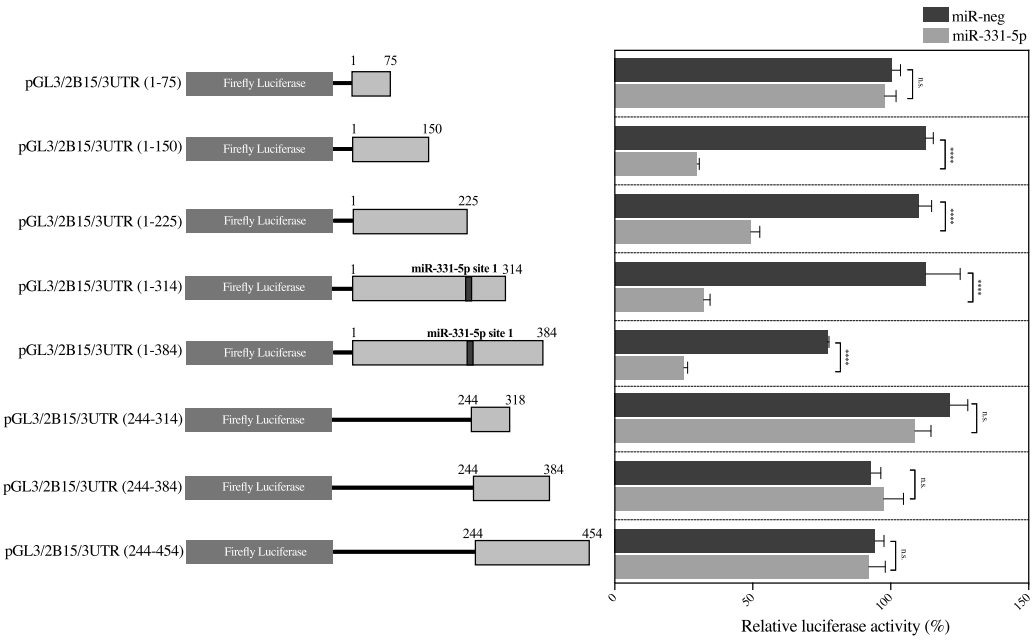

B

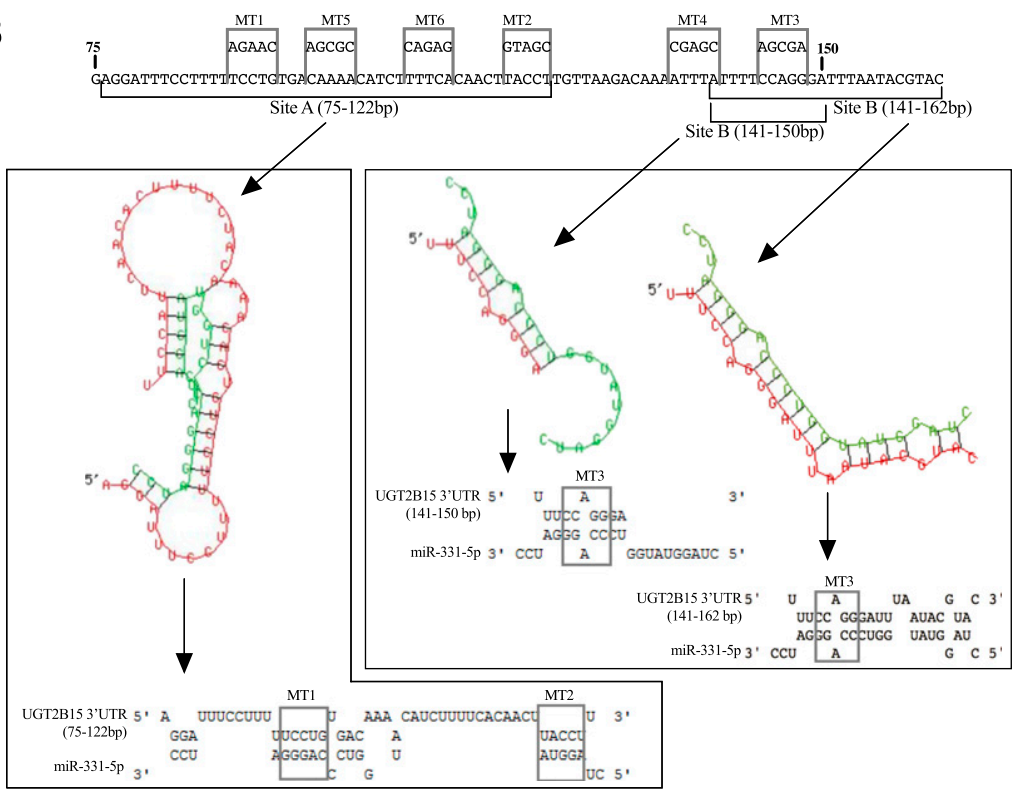

C

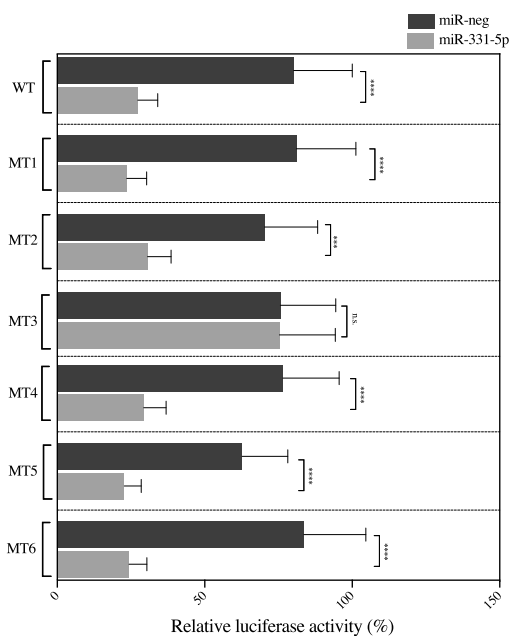

transfected the pGL3/UTR/UGT2B15 nt1-454 luciferase reporter with miR-331-5p, or miR-376c mimics alone, or in combination into LNCaP cells. Both miR-331-5p and miR$376 \mathrm{c}$ mimics significantly reduced reporter activity when transfected alone (by 55\%-65\%); however, cotransfection of
Fig. 4. Discovery of a noncanonical miR-331-5p target site (site 2) in the UGT2B15 3'UTR. (A) A series of eight firefly luciferase reporter constructs containing eight different regions of the UGT2B15 3'UTR (nt1-75, nt1150, nt1-225, nt1-314, nt1-384, nt1-454, nt244-318, and nt244-384) (left diagram) were cotransfected into LNCaP cells with either miR-neg or miR-331-5p mimics, and their activity was measured using luciferase assays as described in Materials and Methods. (B) RNAhybrid predicted two energetically favorable UGT2B15 3'UTR/ miR-331-5p duplex formation sites (sites $A$ and $B$ ) within nucleotides 75-150 of the UGT2B15 3'UTR. Six mutations were created in the pGL3/2B15/3UTR (1-150) reporter construct: mutations MT1, MT2, and MT3 disrupt predicted duplex formation sites; mutations MT4, MT5, and MT6 are in control nonduplex sites. Mutated sites are boxed with the mutated sequences shown above the wild-type sequences. (C) The reporter constructs were cotransfected into LNCaP cells with either miR-neg or miR-331-5p mimics; reporter activities were assessed using luciferase assays as described in Materials and Methods. The activity of the reporter constructs (A and C) was first normalized to the activity of the $\mathrm{pRL}$-null vector and then presented relative to that of pGL3-promoter vector activity (set at a value of $100 \%$ ). Data shown were from two independent experiments performed in quadruplicate; the error bar represents one S.E.M. ${ }^{* * *} P<0.001$. n.s., not statistically significant. these two miRNA mimics further decreased the reporter activity (by $\sim 80 \%$ ) (Fig. 6), indicating synergistic regulation of UGT2B15 3'UTR by miR-331-5p and miR-376c.

UGT2B17 3'UTR Is Not Regulated by miR-331-5p. The sequences of the UGT2B15 and UGT2B17 3'UTRs are 
A

WT

MT A

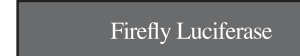

(n)

MT B

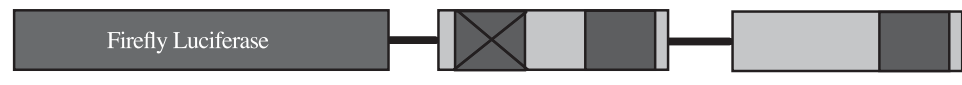

MT C

MT D

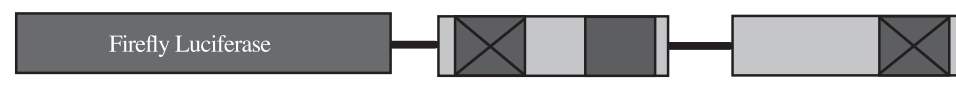

(1)

MT E
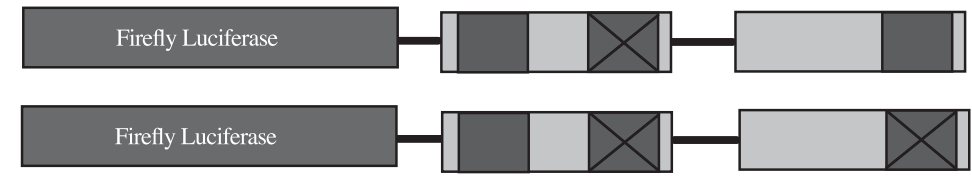

B

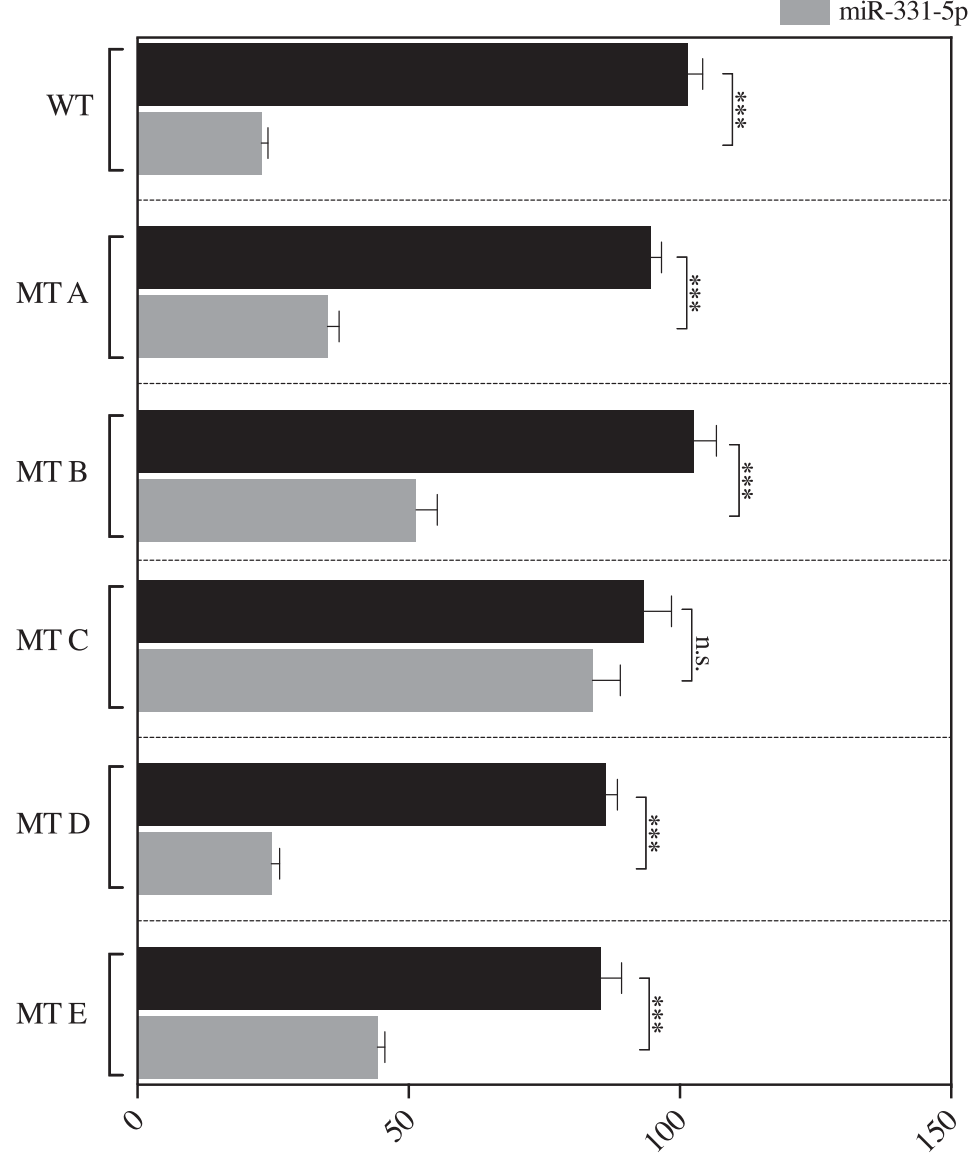

Relative luciferase activity (\%)
Fig. 5. Cooperative regulation of UGT2B15 3'UTR by miR-331-5p via two target sites. (A) Using reporter vectors containing the full-length UGT2B15 3'UTR, the two miR331-5p target sites were mutated [mutation at the seed site of site 1 (MT A) or mutation at either the 3'pairing site (MT B) or seed site (MT D) of site 2] separately or in different combinations (MT C and MT D). (B) Wild-type (WT) or mutated reporter constructs were transfected with either miR-331-5p mimics or miR-neg into LNCaP cells; reporter activities were assessed using luciferase assays as described in Materials and Methods. The activity of the reporter constructs was first normalized to the activity of the pRL-null vector and then presented relative to that of pGL3-promoter vector activity (set at a value of $100 \%$ ). Data shown were from at least two independent experiments performed in quadruplicate; the error bar represents one S.E.M, $* * * P<0.001$. n.s., not statistically significant. highly conserved at their $5^{\prime}$-ends (approximately $210 \mathrm{nt}$ after the TAG codon) but diverge at their $3^{\prime}$-ends (Fig. 1A). The canonical miR-331-5p target site (site 1) lies in the divergent region; hence, the UGT2B17 3'UTR does not contain a site that is equivalent to UGT2B15 miR-331-5p site 1 (Fig. 1A). The noncanonical miR-331-5p site (site 2 ) resides in the region that is conserved between the UGT2B15 and UGT2B17 3'UTRs (Fig. 1A) and a sequence similar to miR-331-5p site 2 can be identified in the UGT2B17 3'UTR. However, transfection of miR-331-5p mimics into LNCaP cells had no impact on UGT2B17 mRNA levels (Fig. 7A), nor on the activity of a luciferase reporter carrying the wild-type UGT2B17 3'UTR 


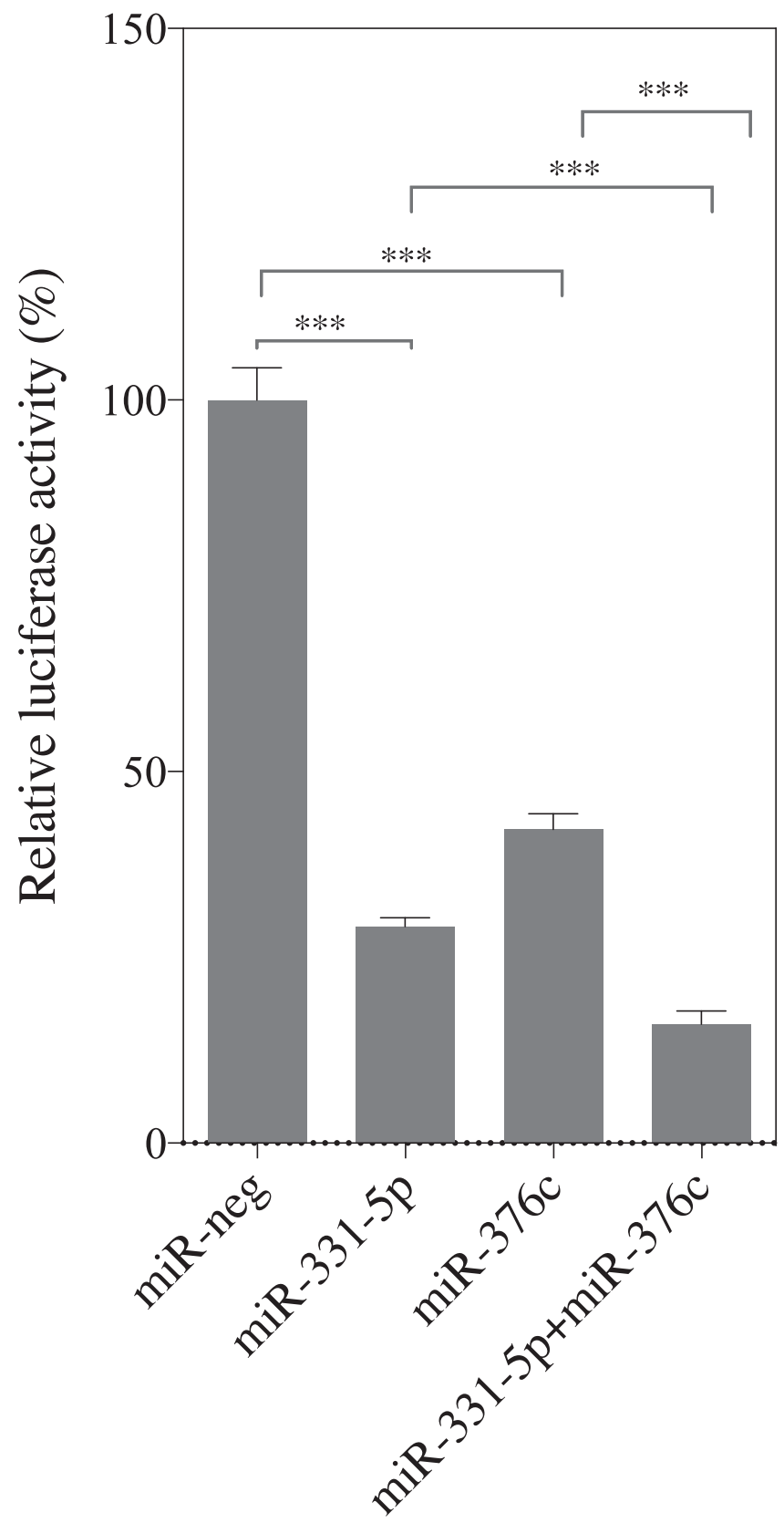

Fig. 6. Cooperative regulation of UGT2B15 3'UTR by miR-331-5p and miR-376c. The firefly luciferase reporter carrying the full-length UGT2B15 3'UTR was transfected into LNCaP cells with miR-neg, miR331-5p, or miR-376c mimics alone or in combination; reporter activities were assessed using luciferase assays as described in Materials and Methods. The activity of the reporter constructs was first normalized to the activity of the pRL-null vector and then presented relative to those of the empty pGL3-promoter vector (set at a value of 100\%). Data shown represent mean \pm S.E.M. from two independent experiments performed in triplicate. ${ }^{* * *} P<0.0001$. n.s., not statistically significant.

(Fig. 7B). This suggests that the putative UGT2B17 miR-331$5 \mathrm{p}$ site 2 is not functional. In support of this observation, RNAhybrid predicted the formation of a miR-331-5p/mRNA duplex $(-14.9 \mathrm{kcal} / \mathrm{mol})$ at this putative site that was much less energetically favorable compared with that $(-22.1 \mathrm{kcal} /-$ mol) formed at the UGT2B15 miR-331-5p site 2 (data not shown). Collectively, these data indicate that the UGT2B17 3'UTR lacks a functional miR-331-5p target site, and is hence not regulated by miR-331-5p.
miR-331-5p and UGT2B15 RNA Levels are Negatively Correlated in Normal Human Tissues and Hepatocellular Carcinoma. We measured miR-331-5p levels in a panel of RNAs from 17 normal human tissues using reversetranscriptase quantitative real-time polymerase chain reaction (Fig. 8A); UGT2B15 mRNA levels in the same tissue panel were recently reported (Wijayakumara et al., 2015). Spearman's correlation analysis showed that miR-331-5p and UGT2B15 mRNA levels were significantly inversely correlated across the tissues (rho $=-0.4952 ; P<0.05$ ) (Fig. 8B), suggesting that miR-331-5p may be involved in repression of UGT2B15 in a number of tissues in vivo. Liver had the highest UGT2B15 mRNA levels (Wijayakumara et al., 2015) and the lowest miR-331-5p levels (Fig. 8A); moreover, analysis of the TCGA hepatocellular carcinoma RNA- and miRNA-seq data sets (TCGA-LIHC, 371 tissues) showed a significant inverse correlation between miR-331 and UGT2B15 levels (rho $=-0.171$, $P=0.0009$ ) (Fig. 8C). Similar analysis of the TCGA-PRAD cohort (498 tissues) also suggested an inverse correlation between levels of miR-331 and UGT2B15, but this correlation was not statistically significant (rho $=-0.043, P=0.335$ ) (Supplemental Fig. 1).

\section{Discussion}

This study identifies the novel mechanism by which miR331-5p regulates UGT2B15. Specifically, we find that repression by this miRNA requires two target sites, one of which mediates canonical seed-based interactions (site 1) and the other mediating noncanonical 3 ' pairing (site 2). Deletion of the seed sequence of site 1 was recently shown to reduce, but not abolish, the ability of $\mathrm{miR}-331-5 \mathrm{p}$ to repress a reporter construct carrying the UGT2B15 3'UTR in LNCaP cells (Margaillan et al., 2016). Our discovery and characterization of the neighboring noncanonical miR-331-5p site (site 2) provides an explanation for the continued ability of miR-331$5 p$ to repress the UGT2B15 3'UTR in the absence of site 1 . Moreover, our study provides a clear example of cooperative regulation by two miRNA target sites.

Canonical miRNA sites contain four types of seed-matched sites (the 6mer, 7mer-m8, 7mer-A1, and 8mer sites) (Brennecke et al., 2005; Lewis et al., 2005; Friedman et al., 2009). An 8mer site is defined by a perfect match at the 6-nt miRNA seed supplemented by an A at miRNA nucleotide 1 and WatsonCrick match to miRNA nucleotide 8 . According to this definition, the UGT2B15 miR-331-5p site 1 is an 8mer site (Fig. 1A). Additional Watson-Crick pairing at miRNA nucleotides 12-17 is believed to enhance miRNA targeting of $7 \mathrm{mer}$ and $8 \mathrm{mer}$ sites (Grimson et al., 2007); these types of $7 \mathrm{mer}$ and $8 \mathrm{mer}$ sites are termed 3' supplementary seed sites (Grimson et al., 2007; Friedman et al., 2009). The 8mer UGT2B15 miR-331-5p site 1 pairs to miR-331-5p at nucleotides $13-15$, and hence corresponds to a typical 3 ' supplementary 8 mer site. Noncanonical miRNA target sites generally contain centered sites and $3^{\prime}$ compensatory sites (Shin et al., 2010). A 3' compensatory site is defined by extensive $3^{\prime}$ pairing in the context of an imperfect seed match (Friedman et al., 2009). The UGT2B15 miR-331-5p target site 2 shows a 1-bp mismatched seed site and an extensive $3^{\prime}$ pairing to miR-331-5p, and hence corresponds to a typical $3^{\prime}$ compensatory site (Fig. 1A). Recent genome-wide crosslinking immunoprecipitation/ RNA-sequencing studies have shown that around 40\%$60 \%$ of miRNA binding sites are noncanonical and include 

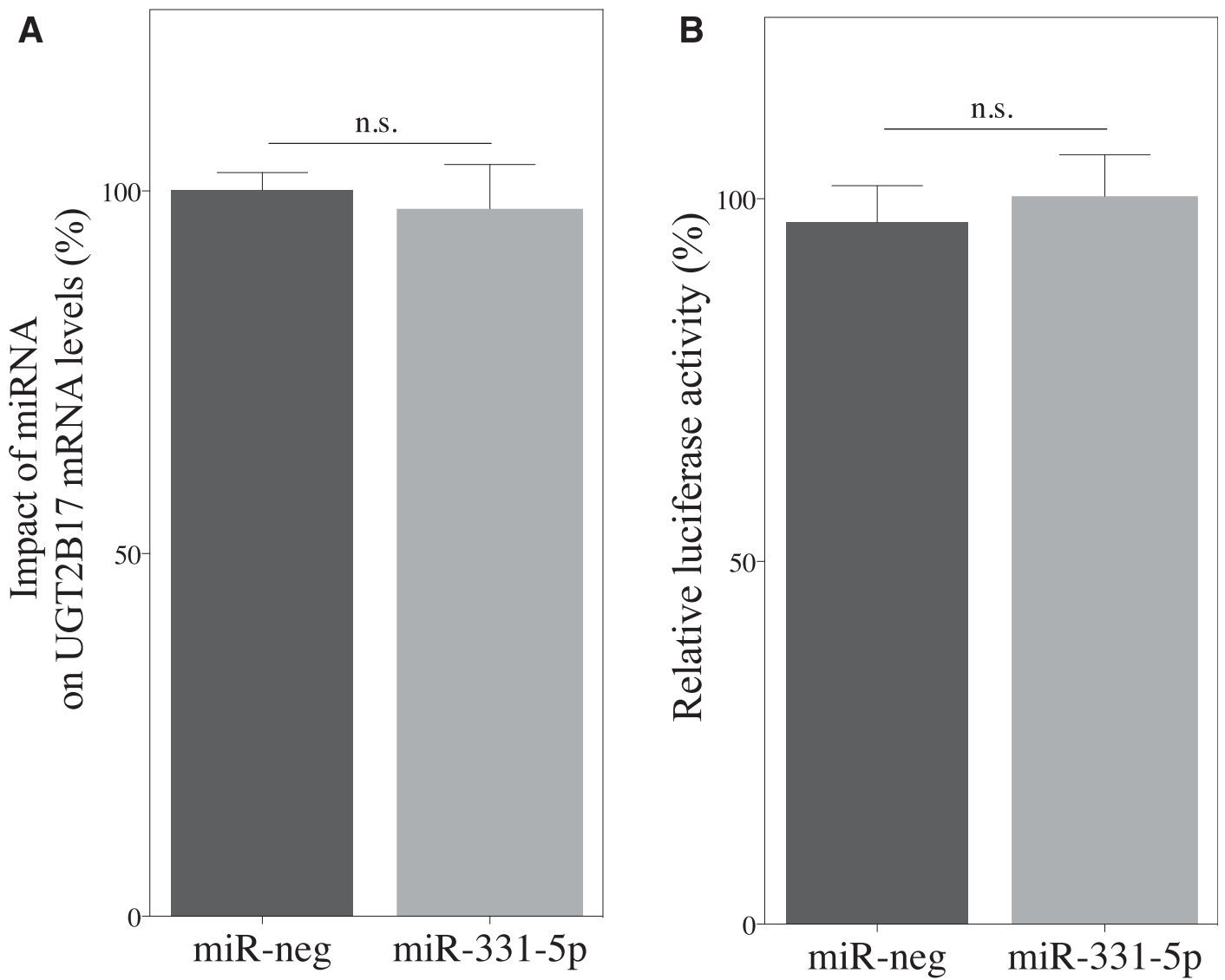

Fig. 7. UGT2B17 3'UTR is not regulated by miR-331-5p. (A) LNCaP cells were transfected with miR-331-5p or miR-neg at $30 \mathrm{nM}$ in triplicate and RNA was extracted from the cells and then subjected to reverse-transcriptase quantitative real-time polymerase chain reaction to measure UGT2B17 mRNA levels. After normalization to the $\beta$-actin mRNA levels, the UGT2B17 mRNA levels in cells transfected with miR-331-5p are presented relative to those in miR-neg-transfected cells (set at a value of 100\%). (B) The firefly luciferase reporter carrying the full-length UGT2B17 $3^{\prime}$ UTR was transfected into LNCaP cells with miR-neg or miR-331-5p; reporter activities were assessed using luciferase assays as described in Materials and Methods. The activity of the reporter constructs was first normalized to the activity of the pRL-null vector and then presented relative to those of the empty pGL3-promoter vector (set at a value of $100 \%$ ). Data shown represent mean \pm S.E.M. from at least two independent experiments performed in triplicate. n.s., not statistically significant.

centered sites and 3 ' compensatory sites (Shin et al., 2010; Loeb et al., 2012; Helwak et al., 2013; Martin et al., 2014). For $3^{\prime}$ compensatory sites, the $3^{\prime}$ pairing is believed to help augment the imperfect seed-mediated weak mRNA/miRNA hybridization. In the case of UGT2B15 miR-331-5p site 2, we showed by mutagenesis that disrupting the 3 ' pairing, rather than the seed pairing, effectively abolished regulation of the UGT2B15 3'UTR by miR-331-5p, suggesting that the 3' pairing plays the pivotal role in mRNA/miRNA duplex formation at this noncanonical miRNA-331-5p site. Collectively, these data indicate that post-transcriptional regulation of UGT2B15 3'UTR by miR-331-5p in prostate cancer LNCaP cells is achieved through two target sites: a $3^{\prime}$ supplementary 8 mer site and a $3^{\prime}$ compensatory site.

The human $m i R-331$ gene (12q22), an intergenic miRNA gene, gives rise to two mature miRNAs: miR-331-3p and miR331-5p. miR-331-5p is considered to be a biomarker for Parkinson's disease (Cardo et al., 2013) and facioscapulohumeral muscular dystrophy (Portilho et al., 2015). miR-331-5p is also implicated in a number of cancers including colorectal adenomas (Verma et al., 2015), leukemia (Feng et al., 2011), and lung cancer (Zhan et al., 2017). In this study, miR-331-5p mimics dramatically repressed endogenous UGT2B15 in LNCaP cells. Moreover, miR-331 and UGT2B15 levels were inversely correlated in a tissue panel and hepatocellular carcinoma (TCGA-LIHC) cohort, suggesting that miR-331-5p plays an important role in defining the basal level of UGT2B15 expression in a range of tissues and in liver cancer.

Although miR-331-5p and UGT2B15 levels appeared to be inversely correlated in LNCaP cells (miR-331-5p levels are low and UGT2B15 levels are high), we did not see significant correlation between miR-331 and UGT2B15 levels in a large prostate cancer cohort. UGT2B15 (and UGT2B17) expression is strongly downregulated by androgens (Guillemette et al., 1996, 1997; Chouinard et al., 2007; Bao et al., 2008) in prostate cancer and AR signaling is likely to maintain tight transcriptional control over the UGT2B15 gene. In this context, direct miRNA-mediated regulation might not be expected to play a central role in defining overall UGT2B15 levels. However, adding further complexity to the regulatory model, the miR331 gene generates both miR-331-5p and miR-331-3p; miR331-3p was reported to repress the AR-signaling pathway through targeting the human epidermal growth factor receptor 2 and PI3k/AKT signaling in prostate cancer cells (Epis et al., 2009). This ability of miR-331-3p to repress AR signaling suggests that it might also indirectly modulate UGT2B15 expression at the transcriptional level; moreover, repression of AR activity could potentially increase UGT2B15 


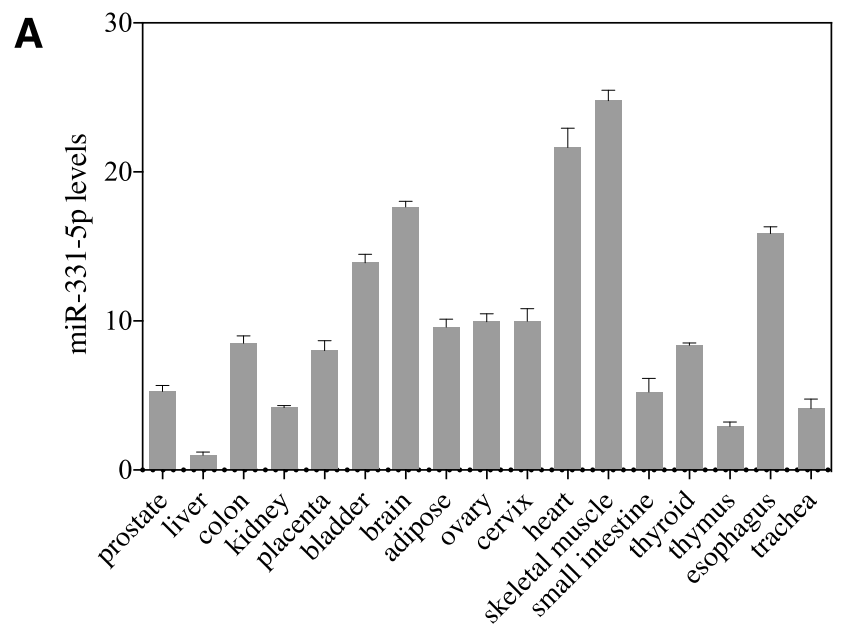

B
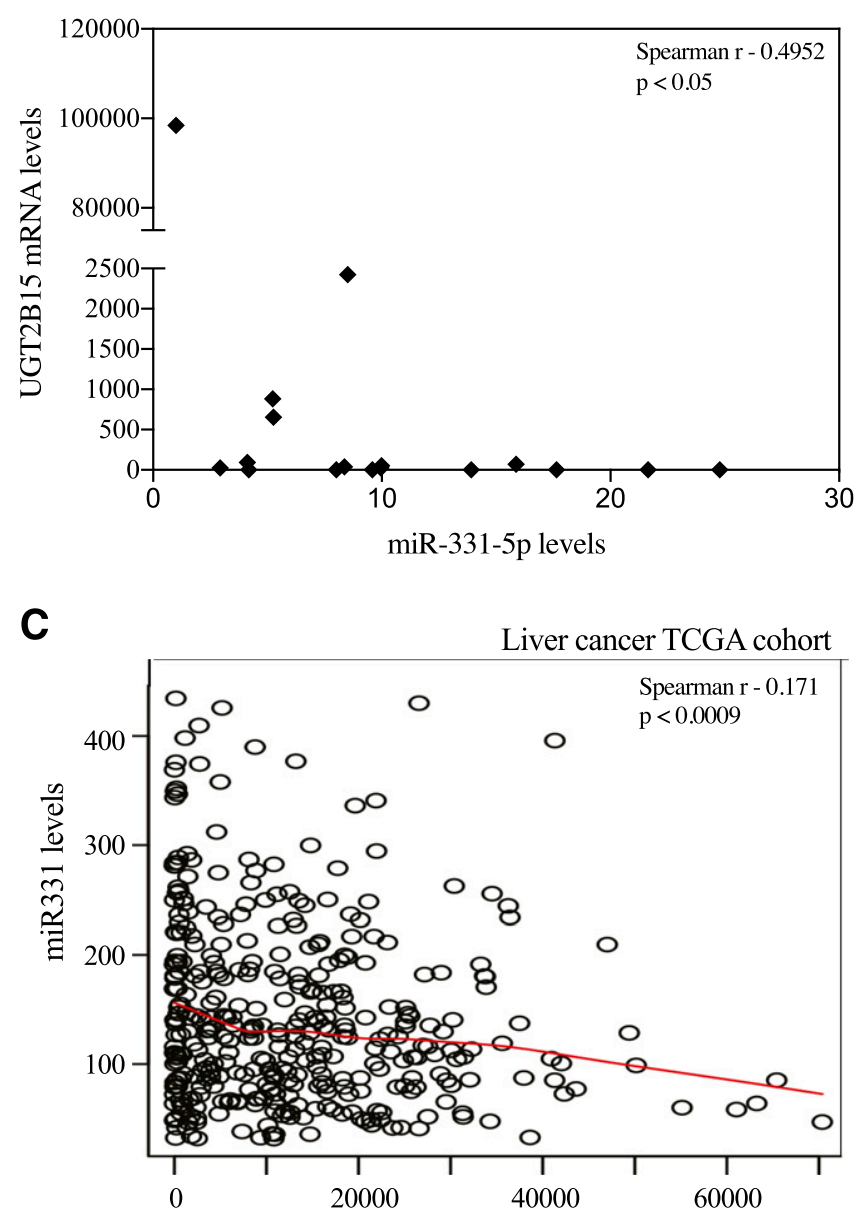

UGT2B15 mRNA levels

Fig. 8. The miR-331-5p and UGT2B15 levels are significantly inversely correlated in a human tissue panel and the TCGA hepatocellular carcinoma cohort. (A) Reverse-transcriptase quantitative real-time polymerase chain reaction analysis of miR-331-5p was conducted using total RNAs from a human tissue panel as described in Materials and Methods. Levels of miR-331-5p were normalized to RNU6-2 and then presented relative to that in liver (set at a value of 1 ). Data shown are from a representative experiment performed in triplicate; the error bar represents \pm S.D. (B) Spearman rank correlation analysis between UGT2B15 mRNA and miR-331-5p levels in the human tissue panel using GraphPad Prism 6 software. (C) Transcriptome profiling data (RNA sequencing and miRNA sequencing) from the TCGA hepatocellular carcinoma cohort (371 samples) were downloaded from the TCGA data portal. Data were transcription. It is tempting to suggest that the lack of a significant correlation between UGT2B15 and miR-331 levels in prostate cancer tissues might reflect the opposing effects of miR-331-5p and miR-331-3p. However understanding the complex network of direct and indirect effects of miR-331derived miRNAs on androgen signaling and UGT2B15 expression will require further study.

Recent studies defined a miR-376c target site at the UGT2B15 3'UTR (Wijayakumara et al., 2015; Margaillan et al., 2016). This miR-376c site is located between the two miR-331-5p sites: 62 bp upstream of miR-331-5p site 2 and $15 \mathrm{bp}$ downstream from miR-331-5p site 1 (Fig. 1A). We demonstrated cooperative regulation of UGT2B15 $3^{\prime}$ UTR by miR-331-5p and miR-376c through these closely located target sites in prostate cancer $\mathrm{LNCaP}$ cells. Similar cooperative regulation of UGT2B4 3'UTR by several miRNAs in liver cancer cell lines via adjacent target sites has been recently reported (Wijayakumara et al., 2017). It remains to be determined whether other $U G T$ genes are also cooperatively regulated by multiple miRNAs.

The proximal UGT2B15 and UGT2B17 promoters have similar sequences and contain highly conserved response elements for the AR and estrogen receptor, and thus they are both downregulated by androgens in prostate cancer cells (Chouinard et al., 2007; Bao et al., 2008) and upregulated by estrogens in breast cancer cells (Hu and Mackenzie, 2009; Hu et al., 2016). The proximal 3'UTRs of UGT2B15 and UGT2B17 also have similar sequences including the highly conserved miR-376c target site, and thus they are both regulated by miR$376 \mathrm{c}$ in prostate cancer cells (Wijayakumara et al., 2015; Margaillan et al., 2016). In this study, we demonstrated negative regulation of UGT2B15 by miR-331-5p via two target sites that are not conserved in the UGT2B17 3'UTR. UGT2B15 miR-331-5p site 1 is located in the distal part of the UGT2B15 $3^{\prime}$ UTR that is highly divergent from that of UGT2B17 (Fig. 1A). In contrast, UGT2B15 miR-331-5p site 2 is located in the proximal part of the UGT2B15 3'UTR that is more highly conserved with UGT2B17. However, the region of the UGT2B17 $3^{\prime}$ UTR that is putatively analogous to UGT2B15 miR-331-5p site 2 shows two nucleotide mismatches when compared with the UGT2B15 sequence; a G/A mismatch in the $3^{\prime}$ pairing sequence of the miR-331-5p target site and a $\mathrm{C} / \mathrm{T}$ mismatch in the seed site (Fig. 1A). We postulate that these nucleotide differences prohibit interactions between the UGT2B17 $3^{\prime}$ UTR and miR331-5p at both the $3^{\prime}$-end and the seed site. Thus, the UGT2B17 3'UTR lacks a functional miR-331-5p target site and is not regulated by miR-331-5p. This provides the first evidence for a posttranscriptional mechanism that can differentially regulate these two important androgen-metabolizing UGTs.

In summary, we report the discovery and characterization of a noncanonical miR-331-5p target site in the UGT2B15 3'UTR and demonstrate that this site cooperates with a neighboring canonical target site (Wijayakumara et al., 2015; Margaillan et al., 2016) to mediate regulation of UGT2B15 by miR-331-5p. Furthermore, we show that miR-331-5p and

normalized and correlation analysis between miR-331 and UGT2B15 mRNA levels was conducted using the Spearman rank method; the graph was drawn using the $\mathrm{R}$ statistical package as described in Materials and Methods. 
miR-376c synergistically regulate the UGT2B15 3'UTR, and that UGT2B17 3'UTR cannot be regulated by miR-331-5p. UGT2B15 and UGT2B17 are major determinants of the androgen response in prostate cancer cells (Chouinard et al., 2007) and also control systemic androgen levels via hepatic metabolism and clearance; defining miRNAs that regulate their expression provides new insights into the mechanisms controlling androgen signaling and may direct novel approaches for prostate cancer therapy.

\section{Acknowledgments}

We thank Dr. Shashi Marri for downloading, processing, and analyzing the TCGA RNA-seq and miRNA-seq data. Dr. Marri was a Research Fellow at the Department of Molecular Medicine and Pathology, Flinders University College of Medicine and Public Health, Flinders Medical Centre, Bedford Park, South Australia, Australia.

\section{Authorship Contributions}

Participated in research design: Wijayakumara, Mackenzie, Hu, Meech.

Conducted experiments: Wijayakumara, $\mathrm{Hu}$.

Performed data analysis: Wijayakumara, Mackenzie, $\mathrm{Hu}$, Meech.

Wrote or contributed to the writing of the manuscript: $\mathrm{Hu}$, Meech, Wijayakumara, Mackenzie, McKinnon.

\section{References}

Balcells I, Cirera S, and Busk PK (2011) Specific and sensitive quantitative RT-PCR of miRNAs with DNA primers. BMC Biotechnol 11:70.

Bao BY, Chuang BF, Wang Q, Sartor O, Balk SP, Brown M, Kantoff PW, and Lee GS (2008) Androgen receptor mediates the expression of UDP-glucuronosyltransferase $2 \mathrm{~B} 15$ and B17 genes. Prostate 68:839-848.

Brennecke J, Stark A, Russell RB, and Cohen SM (2005) Principles of microRNAtarget recognition. PLoS Biol 3:e85.

Cardo LF, Coto E, de Mena L, Ribacoba R, Moris G, Menéndez M, and Alvarez V (2013) Profile of microRNAs in the plasma of Parkinson's disease patients and healthy controls. J Neurol 260:1420-1422.

Chouinard S, Barbier O, and Bélanger A (2007) UDP-glucuronosyltransferase 2B15 (UGT2B15) and UGT2B17 enzymes are major determinants of the androgen response in prostate cancer LNCaP cells. $J$ Biol Chem 282:33466-33474.

Chouinard S, Yueh MF, Tukey RH, Giton F, Fiet J, Pelletier G, Barbier O, and Bélanger A (2008) Inactivation by UDP-glucuronosyltransferase enzymes: the end of androgen signaling. J Steroid Biochem Mol Biol 109:247-253.

Cloonan N (2015) Re-thinking miRNA-mRNA interactions: intertwining issues confound target discovery. BioEssays 37:379-388.

Dluzen DF, Sun D, Salzberg AC, Jones N, Bushey RT, Robertson GP, and Lazarus P (2014) Regulation of UDP-glucuronosyltransferase 1A1 expression and activity by microRNA 491-3p. J Pharmacol Exp Ther 348:465-477.

Dluzen DF, Sutliff AK, Chen G, Watson CJ, Ishmael FT, and Lazarus P (2016) Regulation of UGT2B expression and activity by miR-216b-5p in liver cancer cell lines. J Pharmacol Exp Ther 359:182-193.

Epis MR, Giles KM, Barker A, Kendrick TS, and Leedman PJ (2009) miR-331-3p regulates ERBB-2 expression and androgen receptor signaling in prostate cancer. $J$ Biol Chem 284:24696-24704.

Feng DD, Zhang H, Zhang P, Zheng YS, Zhang XJ, Han BW, Luo XQ, Xu L, Zhou H, Qu LH, et al. (2011) Down-regulated miR-331-5p and miR-27a are associated with chemotherapy resistance and relapse in leukaemia. J Cell Mol Med 15:2164-2175.

Friedman RC, Farh KK, Burge CB, and Bartel DP (2009) Most mammalian mRNAs are conserved targets of microRNAs. Genome Res 19:92-105.

Grimson A, Farh KK, Johnston WK, Garrett-Engele P, Lim LP, and Bartel DP (2007) MicroRNA targeting specificity in mammals: determinants beyond seed pairing. Mol Cell 27:91-105.

Guillemette C, Hum DW, and Bélanger A (1996) Regulation of steroid glucuronosyltransferase activities and transcripts by androgen in the human prostatic cancer LNCaP cell line. Endocrinology 137:2872-2879.

Guillemette C, Lévesque E, Beaulieu M, Turgeon D, Hum DW, and Bélanger A (1997) Differential regulation of two uridine diphospho-glucuronosyltransferases, UGT2B15 and UGT2B17, in human prostate LNCaP cells. Endocrinology 138: 2998-3005.

He L and Hannon GJ (2004) MicroRNAs: small RNAs with a big role in gene regulation. Nat Rev Genet 5:522-531.

Heinlein CA and Chang C (2004) Androgen receptor in prostate cancer. Endocr Rev 25:276-308.

Helwak A, Kudla G, Dudnakova T, and Tollervey D (2013) Mapping the human miRNA interactome by CLASH reveals frequent noncanonical binding. Cell 153: $654-665$.
Hu DG, Gardner-Stephen D, Severi G, Gregory PA, Treloar J, Giles GG, English DR, Hopper JL, Tilley WD, and Mackenzie PI (2010) A novel polymorphism in a forkhead box A1 (FOXA1) binding site of the human UDP glucuronosyltransferase 2B17 gene modulates promoter activity and is associated with altered levels of circulating androstane-3 $3,17 \beta$-diol glucuronide. Mol Pharmacol 78:714-722.

Hu DG and Mackenzie PI (2009) Estrogen receptor $\alpha$, Fos-related antigen-2, and c-Jun coordinately regulate human UDP glucuronosyltransferase $2 \mathrm{~B} 15$ and $2 \mathrm{~B} 17$ expression in response to 17 $\beta$-estradiol in MCF-7 cells. Mol Pharmacol 76:425-439. Hu DG and Mackenzie PI (2010) Forkhead box protein A1 regulates UDPglucuronosyltransferase $2 \mathrm{~B} 15$ gene transcription in $\mathrm{LNCaP}$ prostate cancer cells. Drug Metab Dispos 38:2105-2109.

Hu DG, Meech R, McKinnon RA, and Mackenzie PI (2014) Transcriptional regulation of human UDP-glucuronosyltransferase genes. Drug Metab Rev 46:421-458.

Hu DG, Selth LA, Tarulli GA, Meech R, Wijayakumara D, Chanawong A, Russell R, Caldas C, Robinson JL, Carroll JS, et al. (2016) Androgen and estrogen receptors in breast cancer coregulate human UDP-glucuronosyltransferases 2B15 and 2B17. Cancer Res 76:5881-5893.

Kaarbø M, Klokk TI, and Saatcioglu F (2007) Androgen signaling and its interactions with other signaling pathways in prostate cancer. BioEssays 29:1227-1238.

Kaeding J, Bélanger J, Caron P, Verreault M, Bélanger A, and Barbier O (2008a) Calcitrol $\left(1 \alpha, 25\right.$-dihydroxyvitamin $\left.\mathrm{D}_{3}\right)$ inhibits androgen glucuronidation in prostate cancer cells. Mol Cancer Ther 7:380-390.

Kaeding J, Bouchaert E, Bélanger J, Caron P, Chouinard S, Verreault M, Larouche O, Pelletier G, Staels B, Bélanger A, et al. (2008b) Activators of the farnesoid X receptor negatively regulate androgen glucuronidation in human prostate cancer LNCAP cells. Biochem J 410:245-253.

Krüger J and Rehmsmeier M (2006) RNAhybrid: microRNA target prediction easy, fast and flexible. Nucleic Acids Res 34:W451-W454

Lewis BP, Burge CB, and Bartel DP (2005) Conserved seed pairing, often flanked by adenosines, indicates that thousands of human genes are microRNA targets. Cell 120:15-20.

Livak KJ and Schmittgen TD (2001) Analysis of relative gene expression data using real-time quantitative PCR and the $2^{-\Delta \Delta C T}$ method. Methods 25:402-408.

Loeb GB, Khan AA, Canner D, Hiatt JB, Shendure J, Darnell RB, Leslie CS, and Rudensky AY (2012) Transcriptome-wide miR-155 binding map reveals widespread noncanonical microRNA targeting. Mol Cell 48:760-770

Mackenzie PI, Bock KW, Burchell B, Guillemette C, Ikushiro S, Iyanagi T, Miners JO, Owens IS, and Nebert DW (2005) Nomenclature update for the mammalian UDP glycosyltransferase (UGT) gene superfamily. Pharmacogenet Genomics 15:677-685.

Mackenzie PI, Owens IS, Burchell B, Bock KW, Bairoch A, Bélanger A, FournelGigleux S, Green M, Hum DW, Iyanagi T, et al. (1997) The UDP glycosyltransferase gene superfamily: recommended nomenclature update based on evolutionary divergence. Pharmacogenetics 7:255-269.

Margaillan G, Lévesque É, and Guillemette C (2016) Epigenetic regulation of steroid inactivating UDP-glucuronosyltransferases by microRNAs in prostate cancer. $J$ Steroid Biochem Mol Biol 155:85-93.

Martin HC, Wani S, Steptoe AL, Krishnan K, Nones K, Nourbakhsh E, Vlassov A, Grimmond SM, and Cloonan N (2014) Imperfect centered miRNA binding sites are common and can mediate repression of target mRNAs. Genome Biol 15:R51.

Portilho DM, Alves MR, Kratassiouk G, Roche S, Magdinier F, de Santana EC, Polesskaya A, Harel-Bellan A, Mouly V, Savino W, et al. (2015) miRNA expression in control and FSHD fetal human muscle biopsies. PLoS One 10:e0116853.

Shin C, Nam JW, Farh KK, Chiang HR, Shkumatava A, and Bartel DP (2010) Expanding the microRNA targeting code: functional sites with centered pairing. Mol Cell 38:789-802.

Shukla GC, Singh J, and Barik S (2011) MicroRNAs: processing, maturation, target recognition and regulatory functions. Mol Cell Pharmacol 3:83-92.

Turgeon D, Carrier JS, Lévesque E, Hum DW, and Bélanger A (2001) Relative enzymatic activity, protein stability, and tissue distribution of human steroidmetabolizing UGT2B subfamily members. Endocrinology 142:778-787.

Uchaipichat V, Mackenzie PI, Guo XH, Gardner-Stephen D, Galetin A, Houston JB and Miners JO (2004) Human UDP-glucuronosyltransferases: isoform selectivity and kinetics of 4-methylumbelliferone and 1-naphthol glucuronidation, effects of organic solvents, and inhibition by diclofenac and probenecid. Drug Metab Dispos 32:413-423.

Verma AM, Patel M, Aslam MI, Jameson J, Pringle JH, Wurm P, and Singh B (2015) Circulating plasma microRNAs as a screening method for detection of colorectal adenomas. Lancet 385 (Suppl 1):S100.

Wijayakumara DD, Hu DG, Meech R, McKinnon RA, and Mackenzie PI (2015) Regulation of human UGT2B15 and UGT2B17 by miR-376c in prostate cancer cell lines. J Pharmacol Exp Ther 354:417-425.

Wijayakumara DD, Mackenzie PI, McKinnon RA, Hu DG, and Meech R (2017) Regulation of UDP-glucuronosyltransferases UGT2B4 and UGT2B7 by microRNAs in liver cancer cells. J Pharmacol Exp Ther 361:386-397.

Zhan JW, Jiao DM, Wang Y, Song J, Wu JH, Wu LJ, Chen QY, and Ma SL (2017) Integrated microRNA and gene expression profiling reveals the crucial miRNAs in curcumin anti-lung cancer cell invasion. Thorac Cancer 8:461-470.

Address correspondence to: Dr. Robyn Meech, Department of Clinical Pharmacology and Flinders Centre for Innovation in Cancer, Flinders University College of Medicine and Public Health, Flinders Medical Centre, Bedford Park, South Australia 5042, Australia. E-mail: robyn.meech@flinders. edu.au 